\title{
Advanced squamous lung carcinoma in a patient experiencing long-term survival following repeated responses to gemcitabine and cisplatin chemotherapy: A case report
}

\author{
MIN YU ${ }^{1 *}$, SHUANG ZHANG ${ }^{2 *}$, YANYING LI ${ }^{1}$, ZHENYU DING $^{1}$ and FENG PENG ${ }^{1,2}$ \\ ${ }^{1}$ Department of Thoracic Oncology; ${ }^{2}$ State Key Laboratory of Biotherapy and Cancer Center, \\ West China Hospital, Sichuan University, Chengdu, Sichuan 610041, P.R. China
}

Received June 26, 2014; Accepted February 27, 2015

DOI: $10.3892 / \mathrm{ol} .2015 .3097$

\begin{abstract}
The cisplatin and gemcitabine regimen is one of the most effective regimens against advanced non-small-cell lung cancer. However, tumors that are initially sensitive to chemotherapy treatment may acquire drug resistance. Excision repair cross complementation 1 gene (ERCC1) is involved in the repair of DNA damage caused by cisplatin, and ribonucleotide reductase M1 subunit (RRM1) is associated with gemcitabine resistance in tumor cells. The current study reports the case of a patient with advanced squamous cell lung carcinoma exhibiting low ERCC1 and RRM1 expression levels, who experienced long-term survival following repeated responses to gemcitabine and cisplatin chemotherapy. This case indicates that selected patients may benefit from multiple courses of gemcitabine and cisplatin chemotherapy, and the sustained clinical benefits suggest that further investigation into individualized therapy is merited.
\end{abstract}

\section{Introduction}

In 2014 , lung cancer was predicted to account for $26 \%$ of all female and $28 \%$ of all male cancer-related mortalities in the USA (1). Among all lung cancers, $~ 85 \%$ are non-small cell lung cancer (NSCLC) and the majority of patients are diagnosed at an advanced stage. Adenocarcinoma and squamous cell carcinoma (SCC) are the most common histological subtypes, accounting for $50 \%$ and $30 \%$ of NSCLC cases, respectively (2). Gemcitabine and carboplatin chemotherapy is a well-tolerated regimen against NSCLC, however, the efficiency rate is only $20-40 \%$ (3).

Correspondence to: Dr Feng Peng, Department of Thoracic Oncology, West China Hospital, Sichuan University, 37 Guo Xue Xiang, Chengdu, Sichuan 610041, P.R. China

E-mail: shuangscu@gmail.com

${ }^{*}$ Contributed equally

Key words: non-small-cell lung cancer, squamous lung carcinoma, gemcitabine, cisplatin
Excision repair cross complementation 1 gene (ERCC1) is involved in the repair of DNA damage caused by cisplatin, indicating an association between ERCC1 expression and cisplatin sensitivity. Furthermore, a previous study reported that ERCC1 expression levels were negatively correlated with cisplatin efficacy (4). Ribonucleotide reductase converts ribonucleotide 5'-diphosphate to deoxyribonucleotide 5'-diphosphate, which is the key step required for DNA synthesis (5). Ribonucleotide reductase M1 subunit (RRM1) encodes the regulatory subunit of ribonucleotide reductase, which has been identified as a notable candidate biomarker, particularly with regard to the response to gemcitabine $(6,7)$. A previous study indicated that patients with low RRM1 expression in tumor tissue had a greater median survival time (8). Therefore, the detection of ERCC1 and RRM1 may facilitate the selection of individuals or groups that will gain the greatest benefit from chemotherapy.

The current study reports the case of a patient with advanced squamous lung carcinoma harboring low ERCC1 and RRM1 expression levels and treated with multiple lines of chemotherapy.

\section{Case report}

In March 2010, a 37-year-old female presented to the West China Hospital (Chengdu, Sichuan, China) with a mildly productive cough and chest pain. A general examination indicated an enlarged left supraclavicular lymph node, and the patient underwent a chest computed tomography (CT) scan. The results revealed a mass in the basal segment of the left lung, enlarged lymph nodes in the mediastinum and left lung hilum, and multiple small nodes in the left lung (Fig. 1). Biopsy was performed using a bronchofiberscope, and the histological examination indicated squamous cell carcinoma (SCC) of the lung, with typical morphological characteristics (9). Subsequent immunohistochemical evaluation revealed positive staining for epithelial membrane antigen and pancytokeratin, and negative staining for desmin, smooth muscle actin, S-100, vimentin and cluster of differentiation 1a. Fluorescence in situ hybridization indicated that Epstein-Barr virus encoded RNA was negative. An amplication refractory mutation system showed negative results for epidermal growth factor receptor (EGFR) mutation in exons 18, 19, 20 and 21. Real-time polymerase chain reaction 
A
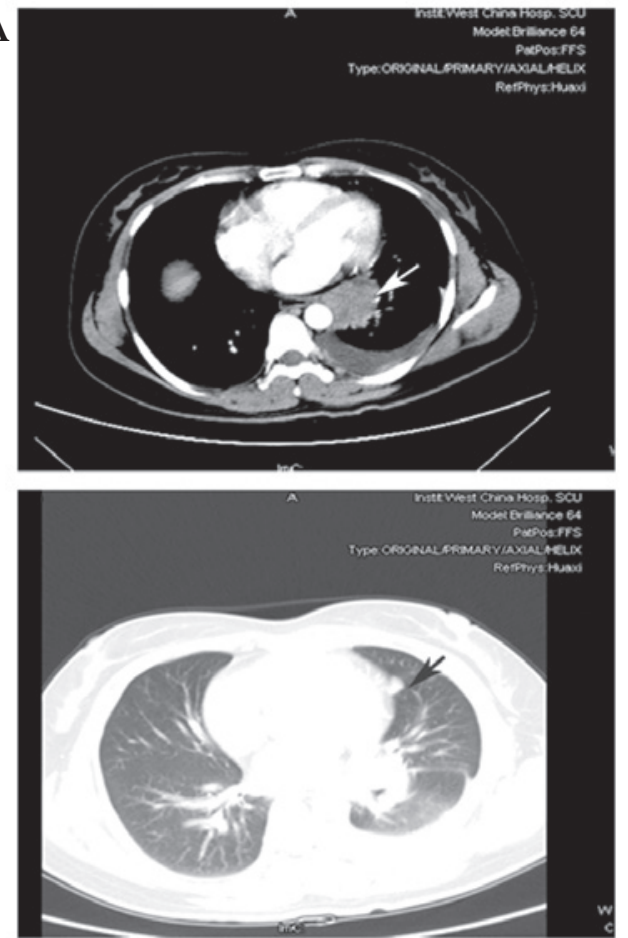

B
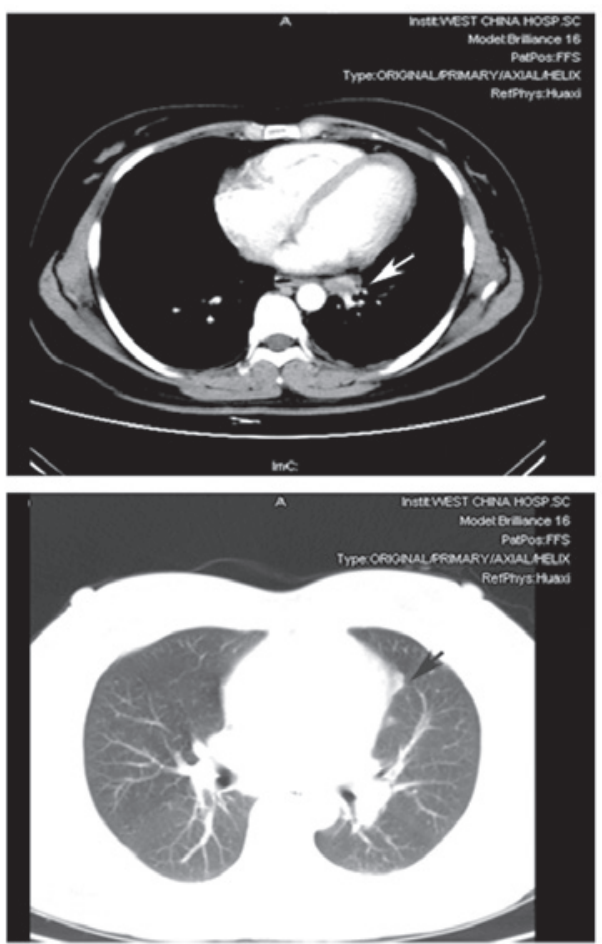

Figure 1. Enhanced chest computed tomography images around the time of first-line cisplatin plus gemcitabine treatment: (A) April 2010, before treatment; and (B) June 2010, after 4 cycles. Arrows indicate the tumor tissue in the basal segment of the left lung and the mediastinal lymph nodes.
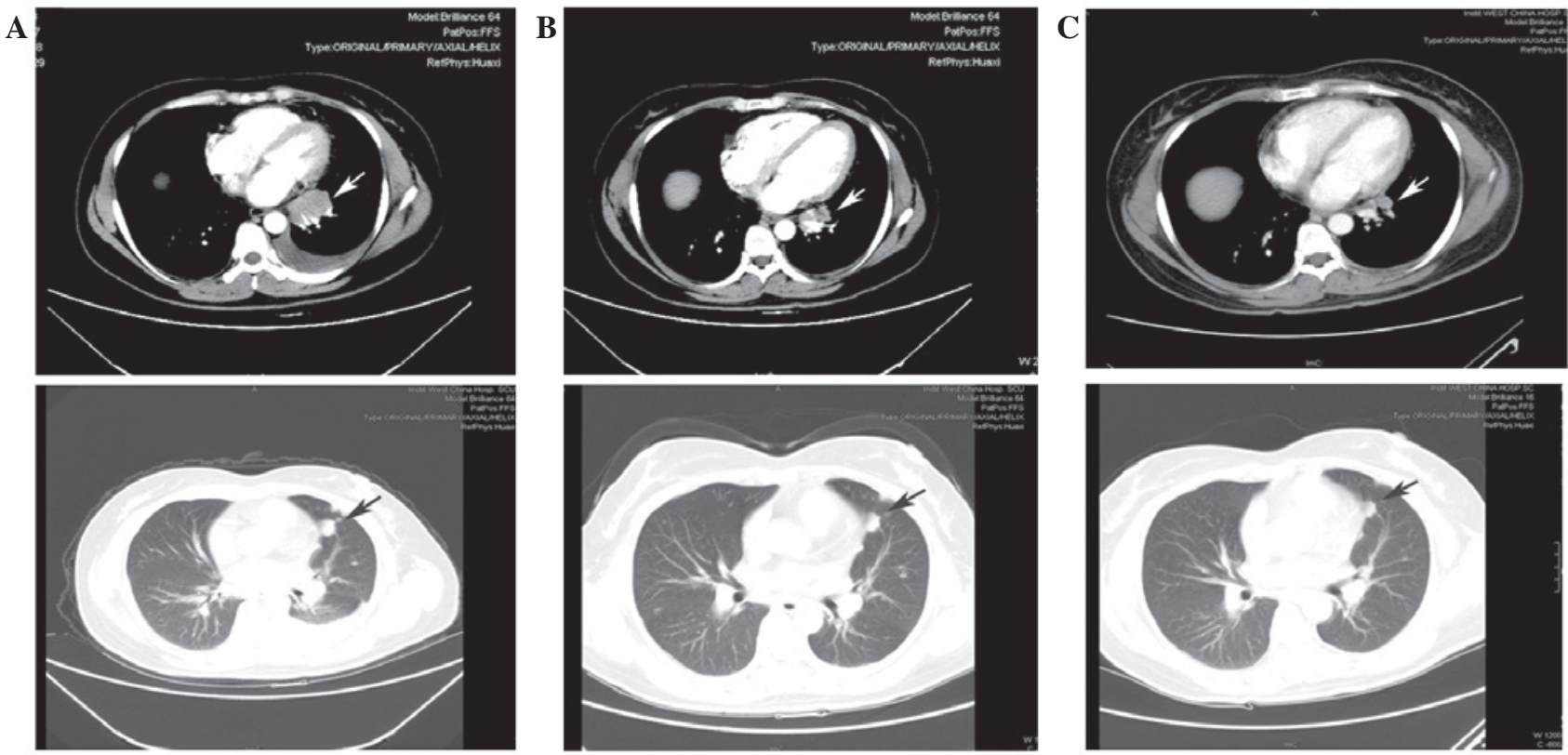

Figure 2. Enhanced chest computed tomography images around the time of third-line cisplatin plus gemcitabine treatment: (A) March 2012, before treatment; (B) May 2012, after 2 cycles; and (C) July 2013, after 4 cycles. Arrows indicate the tumor tissue in the basal segment of the left lung and the mediastinal lymph nodes.

indicated a relative mRNA expression of $6.69 \times 10^{-3}$ for ERCC1 and $8.279 \times 10^{-3}$ for RRM1, which was measured relative to the $\beta$-actin house-keeping gene, as previously described (10). The patient was consequently diagnosed with advanced SCC of the lung (cT4N3Mla, stage IV).

In April 2010, the patient received cisplatin plus gemcitabine (GP) chemotherapy for 2 cycles (gemcitabine, $1,000 \mathrm{mg} / \mathrm{m}^{2}$ days 1 and 8 ; cisplatin, $25 \mathrm{mg} / \mathrm{m}^{2}$, days $1-3$ ), resulting in partial remission (PR). The patient participated in the clinical trial 'Combination chemotherapy with gemcitabine and cisplatin plus recombinant endostatin (endostar) and maintenance therapy with endostar in stage IIIB and IV NSCLC' (registration no. ChiCTR-ONC-08000162), and received 2 cycles of GP regimen, followed by maintenance Endostar for 7 cycles (15 mg, days 1-14; Fig. 1). In March 2011, the patient showed disease progression (PD), based on the increased size 

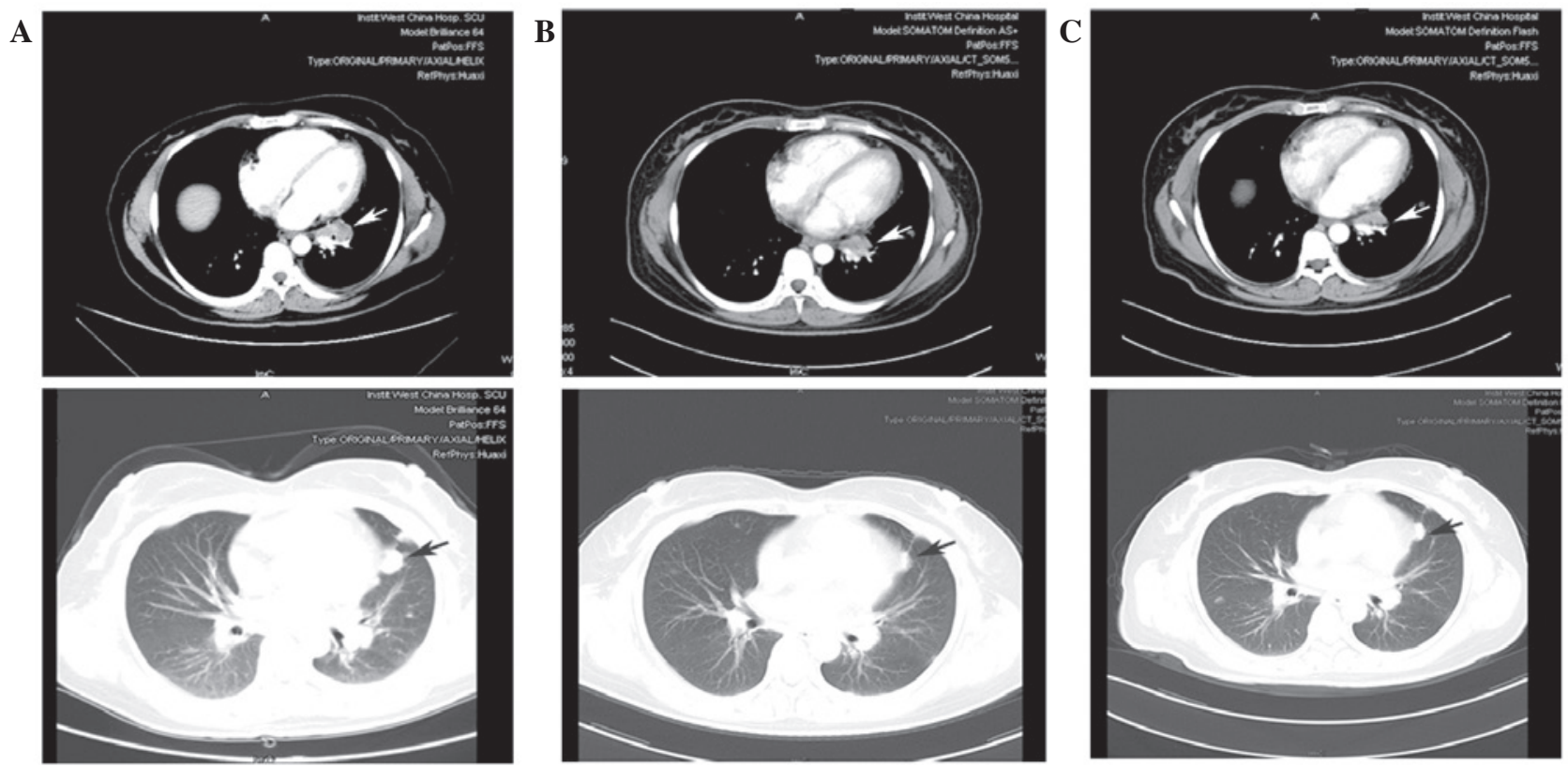

Figure 3. Enhanced chest computed tomography images around the time of fourth-line cisplatin plus gemcitabine treatment: (A) November 2012, before treatment; (B) January 2013, after 2 cycles; and (C) April 2013, after 4 cycles. Arrows indicate the tumor tissue in the basal segment of the left lung and the mediastinal lymph nodes.
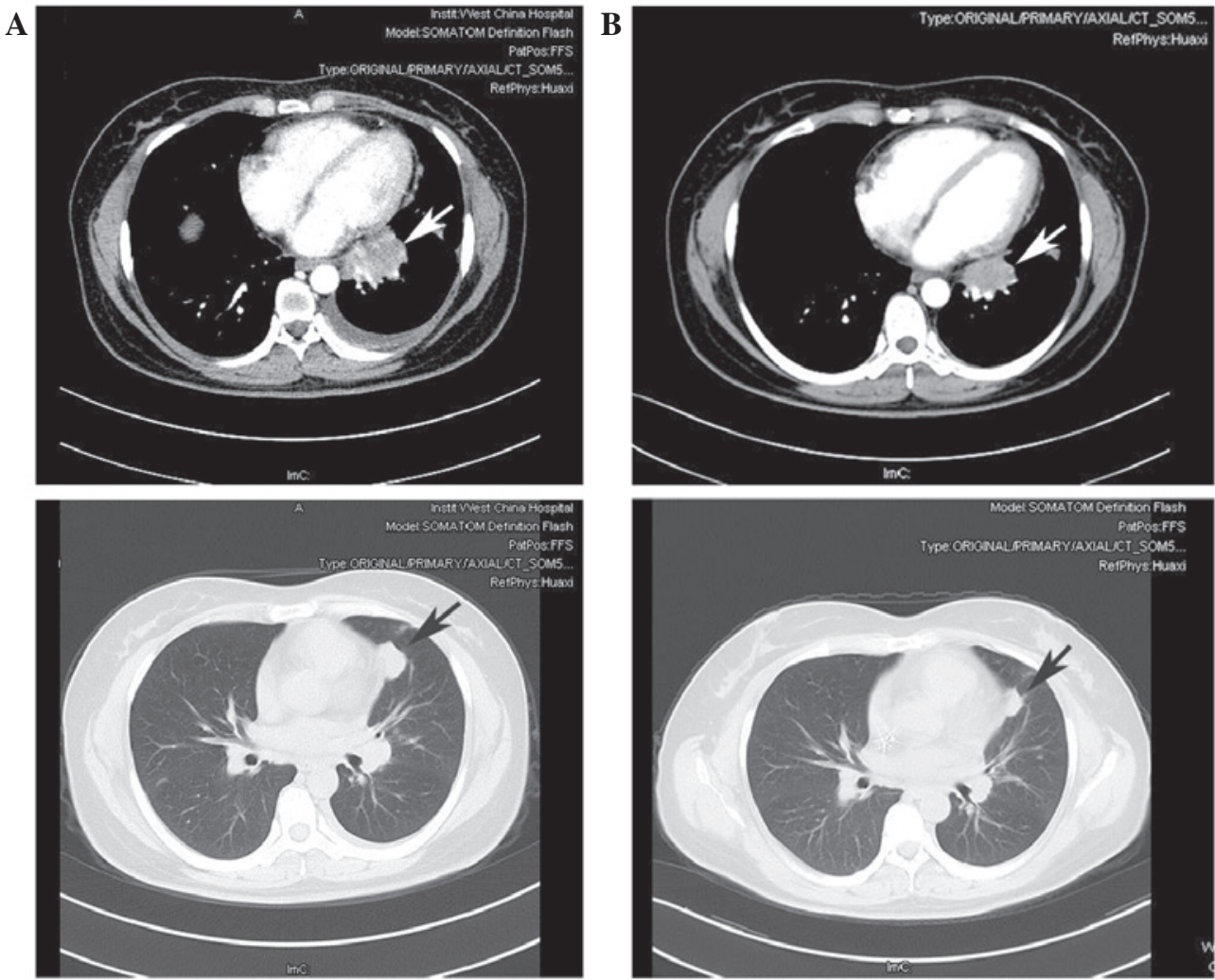

Figure 4. Enhanced chest computed tomography images around the time of sixth-line cisplatin plus gemcitabine treatment: (A) December 2013, before treatment; and (B) March 2014, after 2 cycles. Arrows indicate the tumor tissue in the basal segment of the left lung and the mediastinal lymph nodes.

of the tumor lesions, and commenced a regimen of docetaxel plus oxaliplatin for 5 cycles (docetaxel, $75 \mathrm{mg} / \mathrm{m}^{2}$, day 1 ; oxaliplatin, $130 \mathrm{mg} / \mathrm{m}^{2}$, day 1). In March 2012, the patient presented with a cough and chest pain, and subsequent chest CT imaging revealed that the pulmonary lesions had grown slightly. The patient consented to further GP therapy for 4 cycles, resulting in a PR (Fig. 2). Maintenance gemcitabine was recommended, however, due to poor compliance, the patient refused and instead underwent a 1-month course of erlotinib (150 mg/day), which was self-administered orally. In 
Table I. Summary of therapeutic schedule and effects.

\begin{tabular}{|c|c|c|c|}
\hline Time & Therapeutic schedule & Curative effect & Progression of lung lesions \\
\hline April 2010 to December 2010 & $\begin{array}{l}2 \text { cycles of GP regimen; } \\
2 \text { cycles of GP regimen plus Endostar; } \\
\text { maintenance Endostar for } 7 \text { cycles }\end{array}$ & PR & Growth \\
\hline March 2011 to July 2011 & 5 cycles of DO regimen & $\mathrm{SD}$ & Growth \\
\hline March 2012 to July 2012 & 4 cycles of GP & PR & No growth \\
\hline September 2012 to October 2012 & Erlotinib for 1 month & $\mathrm{PD}$ & Growth \\
\hline November 2012 to March 2013 & 4 cycles of GP regimen & PR & Growth plus new lesions \\
\hline July 2013 to January 2014 & Donafenib for 5 months & $\mathrm{SD}$ & Growth \\
\hline January 2014 to March 2014 & 3 cycles of GP regimen & $\mathrm{SD}$ & - \\
\hline
\end{tabular}

'Growth' refers to an increase in the size of the lesions. GP, cisplatin + gemcitabine; DO, docetaxel + oxaliplatin; PR, partial remission; $\mathrm{SD}$, stable disease; $\mathrm{PD}$, disease progression.

November 2012, enhanced CT imaging revealed that the tumor in the basal segment of the left lung and the lung metastases had increased in size. Due to the previous treatment response, the patient received the GP regimen for 4 cycles, achieving another PR (Fig. 3). In June 2013, enhanced CT imaging revealed that the pulmonary lesions had increased in size and number, and a state of PD was determined. The patient participated in the clinical trial 'Phase I ascending dose tolerance and pharmacokinetic study of Donafenib' (Study ID: 2012L02471). The lung lesions slightly reduced in size, and a stable disease (SD) state was determined. In January 2014, enhanced CT imaging revealed that the tumor and the lung metastases had increased in size. Further GP chemotherapy was administered, and after 2 cycles, the lung lesions were slightly reduced and evaluated as $\mathrm{SD}$ (Fig. 4). Following 4 repeats of the GP regimen, the patient remained alive and was determined to be in an SD state. The patient's disease state will be monitored closely, with follow-up examinations every three months. Due to the sensitivity of this tumor to chemotherapy drugs, the patient has a good prognosis. However, if the tumor progresses in the future, a fifth GP regimen will be adminsitered.

All therapeutic schedules used in the present case and their effects are summarized in Table I. Written informed consent was obtained from the patient for the present study.

\section{Discussion}

In Western countries, approximately one-third of all cancer-related mortalities are caused by lung cancer, and NSCLC accounts for $\sim 80 \%$ of all lung cancer cases, presenting as locally advanced disease in $25-30 \%(1,2)$. Survival is significantly improved by platinum-based chemotherapy in patients with advanced NSCLC. However, it is unclear which platinum-based regimen is the most efficient. A previous meta-analysis, which evaluated a large dataset of 4,556 patients from 13 randomized clinical trials, revealed an increased survival benefit and reduced risk of mortality associated with the gemcitabine-platinum regimen compared with other non-gemcitabine platinum-based regimens in patients with advanced NSCLC (11). Meanwhile, in a phase III study comparing cisplatin plus gemcitabine with cisplatin plus pemetrexed, patients with squamous cell histology showed a significant improvement in survival time with cisplatin/gemcitabine vs. cisplatin/pemetrexed (12). Based on this evidence, the GP regimen was selected for the current patient.

During the treatment course, the GP regimen was administered four times. Each time, the patient benefited following 4 cycles of GP chemotherapy, after which the tumor grew slowly until a PD state was reached. However, the tumors exhibited responses to repeated courses of GP, eventually extending the overall survival time of the patient. In the most recent National Comprehensive Cancer Network (NCCN) guidelines (13), gemcitabine was recommended for second- and third-line therapy, but only for gemcitabine-naïve patients. For SCLC, NCCN guidelines recommended the original effective regimen if disease progression occurred 6 months after schedule completion. However, the application of this principle in NSCLC requires further clinical trial evidence to address whether patients with a good response to first-line treatment who exhibited disease progression after a long time interval may benefit from the original regimen upon repeated administration, how long this time interval may be and whether alternative chemotherapy regimens were more effective than the original regimen. These issues remain unclear, pending further multi-center clinical trials $(14,15)$.

In recent years, research has focused on DNA analysis in cases of drug resistance to inform the individualized treatment of lung cancer. ERCC1 is involved in the repair of DNA damage caused by cisplatin, which suggests an association between ERCC1 expression and cisplatin sensitivity (16). RRM1, encoding the regulatory subunit of ribonucleotide reductase, has been identified as a notable candidate biomarker, particularly with regard to the response to gemcitabine (17). However, a randomized phase III trial did not find statistically significant associations between survival time and RRM1 or ERCC1 levels, thus genetic analysis-based chemotherapy requires further research (18). The methods for testing ERCC1 and RRM1 have not been unified, and the level of expression has no authoritative standard, which presents challenges for RRM1- or ERCC1-guided chemotherapy. In the current case, the expression levels of ERCC1 and 
RRM1 were relatively low, thus repeated GP chemotherapy may increase the efficiency and improve the overall survival of the patient.

EGFR mutation-negative patients are predicted to have a shorter overall survival time, however, the present patient gained long-term survival after repeated responses to GP chemotherapy. In addition to sensitivity to gemcitabine, the patient may also have benefitted from the two clinical trial drugs administered, Endostar and Donafenib. Endostar is a novel recombinant human endostatin, which has been demonstrated to efficiently block angiogenesis and suppress primary tumor growth in clinical trials (19). Compared with bevacizumab, which is administered only for non-squamous NSCLC, Endostar targets the entire tumor endothelial cells and has a broad anticancer spectrum, which includes SCC. Donafenib is an analogue of sorafenib, with the same targets and molecular mechanisms. Similar to adenocarcinoma, SCC exhibits a number of genomic abnormalities, including fibroblast growth factor receptor 1 amplification, MET amplification, BRAF mutation, discoidin domain receptor tyrosine kinase 2 mutation and phosphatidylinositol 3-kinase amplification, which renders it sensitive to existing multi-kinase inhibitors (20). Although the little available pathological tissue was insufficient to allow for further testing of certain rare genetic mutations, Donafenib was effective in the current patient, indicating the existence of cancer-driving genes in the lung SCC.

EGFR mutation-negative NSCLC patients are expected to have a shorter overall survival (21-23), however, the present patient gained a considerable survival benefit through multiple lines of therapy, including the repeated use of effective chemotherapy regimens, anti-angiogenic therapy and multi-kinase inhibitors. In future, individualized therapy based on predictive biomarkers is a promising strategy for the improvement of chemotherapy in patients with advanced NSCLC.

\section{References}

1. Siegel R, Ma J, Zou Z and Jemal A: Cancer statistics, 2014. CA Cancer J Clin 64: 9-29, 2014.

2. Ginsberg MS, Grewal RK and Heelan RT: Lung cancer. Radiol Clin North Am 45: 21-43, 2007.

3. Ceppi P, Volante M, Novello S, et al: ERCC1 and RRM1 gene expressions but not EGFR are predictive of shorter survival in advanced non-small-cell lung cancer treated with cisplatin and gemcitabine. Ann Oncol 17: 1818-1825, 2006.

4. Olaussen KA, Dunant A, Fouret P, et al; IALT Bio Investigators: DNA repair by ERCC1 in non-small-cell lung cancer and cisplatin-based adjuvant chemotherapy. N Engl J Med 355: 983-991, 2006.
5. Jao CY and Salic A: Exploring RNA transcription and turnover in vivo by using click chemistry. Proc Natl Acad Sci USA 105: 15779-15784, 2008.

6. Tiseo M, Bordi P, Bortesi B, et al; Bio-FAST trial group: ERCC1/BRCA1 expression and gene polymorphisms as prognostic and predictive factors in advanced NSCLC treated with or without cisplatin. Br J Cancer 108: 1695-1703, 2013.

7. Su C, Zhou S, Zhang L, et al: ERCC1, RRM1 and BRCA1 mRNA expression levels and clinical outcome of advanced non-small cell lung cancer. Med Oncol 28: 1411-1417, 2011.

8. Rosell R, Danenberg KD, Alberola V, et al; Spanish Lung Cancer Group: Ribonucleotide reductase messenger RNA expression and survival in gemcitabine/cisplatin-treated advanced non-small cell lung cancer patients. Clin Cancer Res 10: 1318-1325, 2004.

9. Travis WD, Brambilla E and Riely GJ: New pathologic classification of lung cancer: relevance for clinical practice and clinical trials. J Clin Oncol 31: 992-1001, 2013.

10. Li Y, Wang LR, Chen J, et al: First-line gemcitabine plus cisplatin in nonsmall cell lung cancer patients. Dis Markers 960458: 2014, 2014.

11. Le Chevalier T, Scagliotti G, Natale R, et al: Efficacy of gemcitabine plus platinum chemotherapy compared with other platinum containing regimens in advanced non-small-cell lung cancer: a meta-analysis of survival outcomes. Lung Cancer 47: 69-80, 2005.

12. Scagliotti GV, Parikh P, von Pawel J, et al: Phase III study comparing cisplatin plus gemcitabine with cisplatin plus pemetrexed in chemotherapy-naive patients with advanced-stage non-small-cell lung cancer. J Clin Oncol 26: 3543-3551, 2008.

13. National Comprehensive Cancer Network. NCCN Clinical Practice Guidelines in Oncology: Non-small cell lung cancer, Version 4.2015.

14. Kalemkerian GP, Akerley W, Bogner P, et al; National Comprehensive Cancer Network: Small cell lung cancer. J Natl Compr Canc Netw 11: 78-98, 2013.

15. Khan K, Hanna GG, Campbell L, et al: Re-challenge chemotherapy with gemcitabine plus carboplatin in patients with non-small cell lung cancer. Chin J Cancer 32: 539-545, 2013.

16. Zheng Z, Chen T, Li X, et al: DNA synthesis and repair genes RRM1 and ERCC1 in lung cancer. N Engl J Med 356: 800-808, 2007.

17. Reynolds C, Obasaju C, Schell MJ, et al: Randomized phase III trial of gemcitabine-based chemotherapy with in situ RRM1 and ERCC1 protein levels for response prediction in non-small-cell lung cancer. J Clin Oncol 27: 5808-5815, 2009.

18. Bepler G, Williams C, Schell MJ, et al: Randomized international phase III trial of ERCC1 and RRM1 expression-based chemotherapy versus gemcitabine/carboplatin in advanced non-small-cell lung cancer. J Clin Oncol 31: 2404-2412, 2013.

19. Wang J, Sun Y and Qin S; Endostar Phase IV Study Group: Results of phase IV clinical trial of combining endostar with chemotherapy for treatment of advanced non-small cell lung cancer (NSCLC). J Clin Oncol 28: 15s, 2010.

20. Perez-Moreno P, Brambilla E, Thomas R and Soria JC: Squamous cell carcinoma of the lung: molecular subtypes and therapeutic opportunities. Clin Cancer Res 18: 2443-2451, 2012.

21. Wu X, Ye Y, Rosell R, et al: Genome-wide association study of survival in non-small cell lung cancer patients receiving platinum-based chemotherapy. J Natl Cancer Inst 103: 817-825, 2011.

22. Cheng L, Alexander RE, Maclennan GT, et al: Molecular pathology of lung cancer: key to personalized medicine. Mod Pathol 25: 347-369, 2012.

23. Pérez-Soler R: Individualized therapy in non-small-cell lung cancer: future versus current clinical practice. Oncogene 28: S38-S45, 2009. 